\title{
The effects of passive leg raising may be detected by the plethysmographic oxygen saturation signal in critically ill patients
}

\author{
Alexandra Beurton ${ }^{1,2^{*}}$, Jean-Louis Teboul ${ }^{1,2}$, Francesco Gavelli ${ }^{1}$, Filipe Andre Gonzalez ${ }^{1}$, Valentina Girotto ${ }^{1}$ \\ Laura Galarza', Nadia Anguel ${ }^{1}$, Christian Richard ${ }^{1}$ and Xavier Monnet ${ }^{1,2}$
}

\begin{abstract}
Background: A passive leg raising (PLR) test is positive if the cardiac index $(\mathrm{Cl})$ increased by $>10 \%$, but it requires a direct measurement of $\mathrm{Cl}$. On the oxygen saturation plethysmographic signal, the perfusion index (PI) is the ratio between the pulsatile and the non-pulsatile portions. We hypothesised that the changes in PI could predict a positive PLR test and thus preload responsiveness in a totally non-invasive way.

Methods: In patients with acute circulatory failure, we measured PI (Radical-7) and Cl (PiCCO2) before and during a PLR test and, if decided, before and after volume expansion (500-mL saline).

Results: Three patients were excluded because the plethysmography signal was absent and 3 other ones because it was unstable. Eventually, 72 patients were analysed. In 34 patients with a positive PLR test (increase in $\mathrm{Cl} \geq 10 \%$ ), $\mathrm{Cl}$ and PI increased during PLR by $21 \pm 10 \%$ and $54 \pm 53 \%$, respectively. In the 38 patients with a negative PLR test, $\mathrm{PI}$ did not significantly change during PLR. In 26 patients in whom volume expansion was performed, $\mathrm{Cl}$ and Pl increased by $28 \pm 14 \%$ and $53 \pm 63 \%$, respectively. The correlation between the $\mathrm{Pl}$ and $\mathrm{Cl}$ changes for all interventions was significant $(r=0.64, p<0.001)$. During the PLR test, if PI increased by $>9 \%$, a positive response of $\mathrm{Cl}(\geq 10 \%)$ was diagnosed with a sensitivity of 91 (76-98\%) and a specificity of 79 (63-90\%) (area under the receiver operating characteristics curve 0.89 (0.80-0.95), $p<0.0001)$.
\end{abstract}

Conclusion: An increase in PI during PLR by $9 \%$ accurately detects a positive response of the PLR test.

Trial registration: ID RCB 2016-A00959-42. Registered 27 June 2016.

Keywords: Fluid responsiveness, Volume expansion, Cardiac index, Perfusion index, Oxygen saturation

\section{Background}

Volume expansion is often the first-line treatment used to increase cardiac index $(\mathrm{CI})$ in patients with acute circulatory failure [1]. However, increasing cardiac preload with fluid administration does not always induce the increase in CI that was expected from it. Moreover, excessive fluid loading with positive cumulative fluid balance may have deleterious effects and impair prognosis of critically ill patients, especially in cases of septic shock $[2,3]$ and acute respiratory distress syndrome $[4,5]$.

\footnotetext{
* Correspondence: alex.beurton@gmail.com

${ }^{1}$ Service de réanimation-médecine intensive, Centre Hospitalier Universitaire de Bicêtre, Hôpitaux universitaires Paris-Sud, Assistance publique - Hôpitaux de Paris, 78, rue du Général Leclerc, F-94 270 Le Kremlin-Bicêtre, France Inserm UMR S_999, Université Paris-Sud, Le Kremlin-Bicêtre, France
}

If preload responsiveness is not obvious, as in the case of fluid loss or at the initial phase of septic shock, it is crucial to predict the response of cardiac output to fluid administration before performing it. The passive leg raising (PLR) test is one of the methods currently available for this purpose [1]. It consists of moving the patient from the semi-recumbent position to a position in which the trunk is horizontal and the inferior limbs are passively elevated at $45^{\circ}$ [6]. The PLR test induces the transfer of some venous blood from the lower part of the body toward the cardiac cavities. It increases the mean systemic pressure [7], resulting in an increase in the pressure gradient of venous return and in $\mathrm{CI}$ in preload responsive patients $[1,8]$. The test has been demonstrated to be reliable by many studies and two

(c) The Author(s). 2019 Open Access This article is distributed under the terms of the Creative Commons Attribution 4.0 International License (http://creativecommons.org/licenses/by/4.0/), which permits unrestricted use, distribution, and 
meta-analyses $[9,10]$. Nevertheless, to detect the changes in CI induced by a PLR test, a direct and real-time measurement of $\mathrm{CI}$ is needed [6], which is often invasive.

The peripheral perfusion index (PI) is derived from the plethysmographic signal of pulse oximetry, which is obtained from the amount of infrared $(940 \mathrm{~nm})$ light transmitted through the vascular bed of a finger. The plethysmographic signal has two components. The pulsatile component reflects changes in the finger blood volume during one cardiac cycle, which may depend on the changes in stroke volume [11], while the non-pulsatile component is related to the light absorbed by the other tissues, such as connective tissue, bone, venous and capillary blood $[12,13]$. Some plethysmographic devices like the Radical-7 (Masimo Corp., Irvine, CA, USA) automatically calculate the ratio of the pulsatile over the non-pulsatile component of the plethysmographic signal, which is called PI and reflects the quality of skin perfusion [12-15]. Then, the changes in the ratio of pulsatile over non-pulsatile component of the plethysmographic signal may depend on the changes in $\mathrm{CI}$.

In this context, monitoring PI might be an attractive method for assessing the effects of the PLR test when no direct measurement of $\mathrm{CI}$ is available. The goal of our study, conducted in critically ill patients with acute circulatory failure, was to test if PI changes could accurately detect a positive response of CI to a PLR test.

\section{Methods}

\section{Patients}

This prospective study was conducted in the 25-bed medical intensive care unit of a university hospital. It was approved by the Institutional Review Board of our institution (Comité pour la Protection des Personnes, Ile-de-France VII, ID RCB: 2016-A00959-42). All patients or their relatives accepted to participate in the study. They were included if they were older than 18 years old, if they were routinely equipped with a PiCCO2 device (Pulsion Medical Systems, Feldkirchen, Germany) and if clinicians in charge decided to perform a PLR test. Fifty $(69 \%)$ patients were included during the resuscitation or stabilisation phase of shock, defined by an increase in the dose of norepinephrine during the last $24 \mathrm{~h}$, and $22(31 \%)$ patients were included at the de-resuscitation phase, defined by a decrease in the dose of norepinephrine over the last $24 \mathrm{~h}$. Patients were excluded $a$ priori if the PLR test was contraindicated (head trauma, deep vein thrombosis in the inferior limbs) or supposed to be unreliable (venous compression stocking, intra-abdominal hypertension, defined as an intra-abdominal pressure (IAP) $>12 \mathrm{mmHg}$ [16]) and a posteriori if the plethysmographic signal was absent and unstable. Plethysmographic signal instability was defined by a precision of $\mathrm{PI} \geq 10 \%$.

\section{PiCCO2 device and haemodynamic variables}

The PiCCO2 system is composed of a central jugular venous catheter and a thermistor-tipped arterial femoral catheter (PC8500, Pulsion Medical Systems, Feldkirchen, Germany) that are connected to a specific device. CI was measured by calibrated pulse contour analysis [17] and by transpulmonary thermodilution [18]. Transpulmonary thermodilution measurements were performed by the injection of cold boluses of $15 \mathrm{~mL}$ of $0.9 \%$ saline into the central venous tract. The average of three consecutive measurements was recorded and averaged [19]. The systemic arterial and central venous pressure curves were recorded continuously by using a data acquisition software (HEM 4.2, Notocord, Croissy-sur-Seine, France). CI was continuously recorded by the PiCCO Win 4.0 software (Pulsion Medical Systems). We measured IAP from the bladder pressure by injecting $25 \mathrm{~mL}$ of saline in the bladder after clamping the urinary drainage bag (AA6118 FOLYSIL, Humlebaek, Denmark). The abdominal pressure transducer was fixed to the patient on the lateral side of the pelvis, $2 \mathrm{~cm}$ below the anterior superior iliac spine. IAP was measured at end-expiration, in the absence of abdominal muscle contractions, which was checked by clinical examination. We defined intra-abdominal hypertension as IAP $\geq 12 \mathrm{mmHg}$ [16].

\section{Perfusion index}

PI was automatically calculated from the plethysmogram by the Radical-7 device as the ratio between the amplitude of the pulsatile and the non-pulsatile components of the light received by the detector of the pulse oximeter, expressed as a percentage. It was measured by a sensor placed on the third or fourth finger, by choosing the one with the highest PI value, as recommended by the constructor. If no signal was obtained on these fingers, we did not attempt to obtain a signal at another site of measurement. The device offers two methods for displaying PI values. With the "short-time" method, PI is displayed in real time with no averaging. With the "long-time" method, the displayed PI values result from a 30-s moving average. We chose the "short-time method" and averaged the PI values over $12 \mathrm{~s}$ because it is the same time that is used by the PiCCO2 device for averaging pulse contour analysis-derived $\mathrm{CI}$ values.

\section{Study design and measurements}

Immediately after inclusion of the patients, when patients were in the semi-recumbent position, we collected demographic characteristics, PI and haemodynamic variables, including heart rate and arterial and central venous pressure. Stroke volume index (SVI) and CI were 
measured by transpulmonary thermodilution. The pressure sensors connected to the arterial and central venous catheters were fixed on the upper arm of the patient at the estimated level of the right atrium. A PLR test was then performed by transferring the patient to the PLR position, in which the lower limbs are passively elevated at $45^{\circ}$ and the trunk is horizontal [6]. When the PLR test had induced its maximal effect on $\mathrm{CI}$, which occurs within $1 \mathrm{~min}$, we performed another set of measurements including CI. At this time, CI was measured by pulse contour analysis, because the effects of PLR may decrease after reaching their maximum in some patients, so that transpulmonary thermodilution may miss the maximal effects because of the time required for performing three boluses injections [6]. Then, we moved the patient back to the semi-recumbent position. We performed a third set of measurements, including heart rate, arterial and central venous pressure, PI, SVI and CI measured by transpulmonary thermodilution.

The PLR test could be planned in view of infusing fluid. In such cases, in case of a positive PLR test, clinicians could decide to perform a volume expansion with $500 \mathrm{~mL}$ of saline, weighting its risks and benefits. The PLR test could also be performed for guiding the decision of fluid removal [20]. In such cases, a negative PLR test could lead to fluid removal, again depending on the decision of the clinicians in charge. Immediately after the end of fluid infusion, we performed the last set of measurements of mean arterial pressure, heart rate, central venous pressure, PI, SVI and CI (transpulmonary thermodilution). Catecholamines and sedative drugs doses as well as ventilation settings were kept constant during the study.

\section{Measurement of the precision of $\mathrm{PI}$}

In each patient, during a period of time when the haemodynamic status was stable (change in pulse contour analysis-derived $\mathrm{CI}<10 \%$ ), we recorded five successive values of PI, each averaged over $12 \mathrm{~s}$. During this time, the position of the plethysmographic sensor was kept unchanged. We calculated the coefficient of variation of PI as being the standard deviation divided by the mean of the five measurements [17-19].

The coefficient of variation is a relative measure of the dispersion of the data around the mean. It allows the comparison of the degree of variation from one sample to another, even if the averages are different. The precision was calculated as being two times the coefficient of variation and the least significant change as the coefficient of variation $\times 1.96 \times \sqrt{ } 2$ [19-21]. The least significant change is the most interesting variable to observe since it indicates the minimum change measured by the device that can be trusted as a real change of measurement [22]. It must be compared to the changes that have been actually observed during the study.

\section{Statistical analysis}

The PLR test was defined as positive if it increased $\mathrm{CI} \geq$ $10 \%$. The response to volume expansion was defined as positive if $\mathrm{CI}$ increased $\geq 15 \%$ just after fluid administration. Data were expressed as mean \pm standard deviation, median [interquartile range, IQR] and number (percentage). Normality was assessed by the Kolmogorov-Smirnov test. Pairwise comparisons of values between different study times were performed by paired Student $t$ tests. Comparisons between patients with positive PLR and patients with negative PLR tests were performed by two-tailed Student $t$ tests or the Wilcoxon test.

We compared the relative changes of CI to those of PI by linear regression analysis (for percent changes). To assess the trending ability of PI, we constructed a regression curve. This allowed the calculation of the percentage of total data points for which the directional changes of PI were concordant with those of CI. Correlations were assessed by the Spearman coefficient. Receiver operating characteristic (ROC) curves (with 95\% confidence interval) were generated for describing the ability of the PLR-induced percent changes in PI to detect the PLR-induced percent changes in CI. The areas under ROC curves were compared by the Hanley-McNeil test [23]. The Youden index was calculated as sensitivity + specificity -1 and was used to determine the diagnostic threshold.

The calculation of the sample size was based on the areas under the ROC curves. Considering a null hypothesis at 0.75, expecting an area under the curve for the PLR-induced changes in PI of 0.90 and taking into account an $\alpha$ risk at $5 \%$ and a $\beta$ risk at $20 \%$, we planned to include 34 patients per group. Statistical analysis was performed using MedCalc 11.6.0 software (Mariakerke, Belgium).

\section{Results}

\section{Patient characteristics}

We initially screened 85 patients which the characteristics are detailed in Table 1. Among the 85 screened patients, 7 were excluded because of intra-abdominal hypertension (IAP, $18 \pm 3 \mathrm{mmHg}$ ). Three other ones were excluded because the plethysmographic signal was not obtained. Their characteristics were not different from the other ones in terms of arterial pressure, dose of norepinephrine or shock origin (septic for 8 patients and hypovolemic for 2). Three patients presented an unstable plethysmography signal. Two of these 3 patients were the only screened ones who presented atrial fibrillation. 
Table 1 Patient characteristics $(n=72)$

\begin{tabular}{|c|c|}
\hline Age (mean $\pm S D$, years) & $64 \pm 13$ \\
\hline Gender (male, $n(\%)$ ) & $56(77 \%)$ \\
\hline Weight (mean $\pm S D, k g)$ & $72 \pm 16$ \\
\hline Height (mean \pm SD, cm) & $168 \pm 10$ \\
\hline SAPS $\|($ mean \pm SD) & $60 \pm 20$ \\
\hline \multicolumn{2}{|l|}{ Type of shock ( $n(\%))$ : } \\
\hline Septic & $51(70 \%)$ \\
\hline Cardiogenic & $12(17 \%)$ \\
\hline Hypovolemic & $9(13 \%)$ \\
\hline \multicolumn{2}{|l|}{ Catecholamines } \\
\hline Norepinephrine (n (\%)) & $52(72 \%)$ \\
\hline $\begin{array}{l}\text { Dose of norepinephrine (median } \\
\text { [interquartile range], } \mu \mathrm{g} / \mathrm{kg} / \mathrm{min} \text { ) }\end{array}$ & $0.5[0.1-0.6]$ \\
\hline Dobutamine (n (\%)) & $8(11 \%)$ \\
\hline $\begin{array}{l}\text { Dose of dobutamine (median } \\
\text { [interquartile range], } \mu \mathrm{g} / \mathrm{kg} / \mathrm{min} \text { ) }\end{array}$ & 16 [14-20] \\
\hline \multicolumn{2}{|l|}{ Respiratory settings } \\
\hline Mechanical ventilation (n (\%)) & $56(78 \%)$ \\
\hline Tidal volume (mean $\pm \mathrm{SD}, \mathrm{mL} / \mathrm{kg}$ of PBW) & $5.8 \pm 1.4$ \\
\hline Plateau pressure $\left(\right.$ mean $\left.\pm \mathrm{SD}, \mathrm{cmH}_{2} \mathrm{O}\right)$ & $23.5 \pm 3.8$ \\
\hline $\begin{array}{l}\text { Positive end-expiratory pressure } \\
\left(\text { mean } \pm \mathrm{SD}, \mathrm{cmH}_{2} \mathrm{O}\right)\end{array}$ & $9.8 \pm 3.5$ \\
\hline
\end{tabular}

Values are expressed as mean \pm standard deviation, number $(n)$ and frequency (\%) or median and interquartile range

PBW predicted body weight, SAPS II Simplified Acute Physiology Score, SD standard deviation

No patient presented frequent atrial or ventricular extrasystoles. Eventually, 72 patients were included. A flow chart is displayed in Additional file 1: Figure S1.

No patient had acute cor pulmonale or severe valvular disease. The IAP was $4 \pm 3 \mathrm{mmHg}$. Among the 20 (28\%) patients who had no norepinephrine at the time of inclusion, it had been stopped in $13(18 \%)$ patients, who were in the stabilisation phase of their disease, and it had never been administered before in 7 (10\%) patients. The lactate level at the time of inclusion was $1.8 \pm 1.1$ $\mathrm{mmol} / \mathrm{L}$. Most of the patients (56 (78\%)) were mechanically ventilated.

\section{PI absolute values}

At baseline, the value of PI was $2.5 \pm 1.9 \%$, ranging from 0.2 to $6.7 \%$. It was lower than $1 \%$ in 23 patients. The value of PI at baseline was correlated with the dose of norepinephrine $(r=-0.29, p=0.04)$. The value of PI was similar in patients with and without norepinephrine infusion $(2.5 \pm 1.9 \%$ vs. $2.2 \pm 1.8 \%$, respectively, $p=0.39)$, as well as in patients with and without mechanical ventilation $(2.5 \pm 1.9 \%$ vs. $1.6 \pm 1.7 \%$, respectively, $p=0.56)$. It was also similar between patients receiving the lowest and the highest doses of norepinephrine, as defined according to its median value $(2.6 \pm 2.0 \%$ vs. $2.6 \pm 2.0 \%$, respectively, $p=0.56$ ) (Table 1 ). The absolute value of PI at different study times was correlated with mean $(r=$ $0.20, p=0.003)$ and with diastolic arterial pressure $(r=$ $0.16, p=0.01$ ).

In the subgroup of patients with $\mathrm{PI} \leq 1 \%$, the dose of norepinephrine was similar as in the other ones $(0.5 \pm$ 0.7 vs. $0.4 \pm 0.3 \mu \mathrm{g} / \mathrm{kg} / \mathrm{min}$, respectively, $p=0.72$ ). These patients with $\mathrm{PI}<1 \%$ did also not differ in terms of lactate level at baseline $(1.9 \pm 1.1$ vs. $1.7 \pm 1.1 \mathrm{mmol} / \mathrm{L}$, respectively, $p=0.49$ ), time elapsed between the onset of shock and the inclusion $(120 \pm 108$ vs. $98 \pm 53 \mathrm{~h}$, respectively, $p=0.44$ ) or Simplified Acute Physiology Score (SAPS) II score (62 \pm 21 vs. $56 \pm 18$, respectively, $p=0.43$ ).

\section{Effects of PLR and volume expansion on PI}

The haemodynamic variables and their time course are reported in Table 2. The changes in CI and PI during a PLR test in a typical fluid responder and a typical non-responder are displayed in Fig. 1. The PLR test was positive (PLR-induced increase in $\mathrm{CI} \geq$ $10 \%)$ in 34 patients. The PI value at baseline was similar in these patients and in the other ones (Table 2). In patients in whom the PLR test was positive, CI, SVI and PI significantly increased during PLR by $21 \pm 10 \%, 18 \pm 19 \%$ and $54 \pm 53 \%$, respectively $(p<0.001$ for both) (Fig. 2). The PLR test was negative (CI increased by $<10 \%)$ in 38 patients. In these patients, CI and SVI significantly increased during PLR by $2 \pm 4 \%$ and $2 \pm 7 \%$, respectively, and PI did not significantly change.

Volume expansion was decided after 27 positive PLR tests. Twenty-six of these patients were eventually fluid responders (fluid-induced increase in $\mathrm{CI} \geq 15 \%$ ). In these patients, after volume expansion, $\mathrm{CI}$ and PI significantly increased by $28 \pm 14 \%$ and $53 \pm 63 \%$, respectively ( $p<$ 0.001 for both). Only 1 patient with a positive PLR test was a fluid non-responder. In this patient, the fluid-induced increase in CI was 9\%, whereas the PLR-induced increase in $\mathrm{CI}$ was $15 \%$.

\section{Ability of PI changes to detect a positive PLR test}

During PLR, if PI increased by $>9 \%$, a positive response of CI (increase by $>10 \%$ ) to PLR could be diagnosed with a sensitivity of $91 \%$ (76-98\%), a specificity of $79 \%$ (63-90\%), a positive predictive value of $80 \%$ (64-91\%) and a negative predictive value of $91 \%(76-98 \%)$. The area under the ROC curve was $0.89(0.80-0.95)(p<$ 0.0001 vs. 0.5 ) (Fig. 3). PI increased by $>9 \%$ in 31 patients under 34 with a positive PLR.

In the subgroup of patients with $\mathrm{PI} \leq 1 \%$, the area under the ROC curve was similar to the area under the ROC curve in patients with PI $>1 \%((0.94 \quad(0.75-0.99)$ vs. $0.88(0.77-0.96)$, respectively, $p=0.48)$. 
Table 2 Haemodynamic variables

\begin{tabular}{|c|c|c|c|c|}
\hline & Baseline 1 & PLR test & Baseline 2 & After volume expansion \\
\hline \multicolumn{5}{|l|}{ Heart rate (beats/min) } \\
\hline - Positive PLR test $(n=34)$ & $94 \pm 16$ & $95 \pm 20$ & $97 \pm 17$ & \multirow[t]{2}{*}{$93 \pm 15^{\dagger}$} \\
\hline - Negative PLR test $(n=38)$ & $90 \pm 20$ & $89 \pm 20$ & $88 \pm 20$ & \\
\hline \multicolumn{5}{|l|}{ Systolic arterial pressure (mmHg) } \\
\hline - Positive PLR test $(n=34)$ & $115 \pm 23$ & $128 \pm 29^{*}$ & $112 \pm 28$ & \multirow[t]{2}{*}{$122 \pm 34^{\dagger}$} \\
\hline - Negative PLR test $(n=38)$ & $125 \pm 20$ & $130 \pm 21^{*}$ & $123 \pm 20$ & \\
\hline \multicolumn{5}{|c|}{ Diastolic arterial pressure $(\mathrm{mmHg})$} \\
\hline - Positive PLR test $(n=34)$ & $58 \pm 11$ & $63 \pm 10^{*}$ & $57 \pm 13$ & \multirow[t]{2}{*}{$61 \pm 15^{\dagger}$} \\
\hline - Negative PLR test $(n=38)$ & $60 \pm 9$ & $63 \pm 9^{*}$ & $59 \pm 9$ & \\
\hline \multicolumn{5}{|l|}{ Mean arterial pressure (mmHg) } \\
\hline - Positive PLR test $(n=34)$ & $77 \pm 13$ & $87 \pm 21^{*}$ & $75 \pm 17$ & \multirow[t]{2}{*}{$80 \pm 19^{\dagger}$} \\
\hline - Negative PLR test $(n=38)$ & $81 \pm 12$ & $85 \pm 13^{*}$ & $80 \pm 12$ & \\
\hline \multicolumn{5}{|l|}{ Central venous pressure (mmHg) } \\
\hline - Positive PLR test $(n=34)$ & $10 \pm 5$ & $13 \pm 5^{*}$ & $10 \pm 4$ & \multirow[t]{2}{*}{$11 \pm 5^{\dagger}$} \\
\hline - Negative PLR test $(n=38)$ & $10 \pm 5$ & $14 \pm 5^{*}$ & $9 \pm 5$ & \\
\hline \multicolumn{5}{|l|}{ Cardiac index $\left(\mathrm{L} / \mathrm{min} / \mathrm{m}^{2}\right)$} \\
\hline - Positive PLR test $(n=34)$ & $3.38 \pm 1.21$ & $4.03 \pm 1.31^{*}$ & $3.20 \pm 1.20$ & \multirow[t]{2}{*}{$4.02 \pm 1.35^{\dagger}$} \\
\hline - Negative PLR test $(n=38)$ & $3.19 \pm 1.26$ & $3.26 \pm 1.32^{* *}$ & $3.15 \pm 1.31$ & \\
\hline \multicolumn{5}{|l|}{$\operatorname{GEDV}\left(\mathrm{mL} / \mathrm{m}^{2}\right)$} \\
\hline - Positive PLR test $(n=34)$ & $766 \pm 165$ & & $768 \pm 205$ & \multirow[t]{2}{*}{$789 \pm 127^{\dagger}$} \\
\hline - Negative PLR test $(n=38)$ & $800 \pm 242$ & & $792 \pm 219$ & \\
\hline \multicolumn{5}{|l|}{$\mathrm{SVI}\left(\mathrm{mL} / \mathrm{m}^{2}\right)$} \\
\hline - Positive PLR test $(n=34)$ & $37 \pm 13$ & $44 \pm 15^{*}$ & $36 \pm 12$ & \multirow[t]{2}{*}{$43 \pm 15^{\dagger}$} \\
\hline - Negative PLR test $(n=38)$ & $37 \pm 15$ & $38 \pm 16$ & $37 \pm 17$ & \\
\hline \multicolumn{5}{|l|}{$\operatorname{PPV}(\%)^{\ddagger}$} \\
\hline - Positive PLR test $(n=28)$ & $10.0 \pm 4.0$ & & $9.8 \pm 5.9$ & \multirow[t]{2}{*}{$11.9 \pm 16.2$} \\
\hline - Negative PLR test $(n=28)$ & $8.0 \pm 5.1$ & & $8.5 \pm 4.7$ & \\
\hline \multicolumn{5}{|l|}{ PI (\%) } \\
\hline - Positive PLR test $(n=34)$ & $2.9 \pm 2.0$ & $4.1 \pm 2.3^{*}$ & $2.1 \pm 1.4$ & \multirow[t]{2}{*}{$3.0 \pm 1.9^{\dagger}$} \\
\hline - Negative PLR test $(n=38)$ & $2.0 \pm 1.8$ & $2.0 \pm 2.0^{*}$ & $2.1 \pm 1.9$ & \\
\hline
\end{tabular}

Values are expressed as mean \pm standard deviation

$P L R$ passive leg raising test, GEDV global end-diastolic volume, SVI stroke volume index, PPV pulse pressure variation, $P I$ perfusion index ${ }^{*} p<0.05$ vs baseline 1

${ }^{+} p<0.05$ vs baseline 2

$p<0.05$ between positive and negative PLR test

${ }^{\ddagger}$ In mechanically ventilated patients

\section{Ability of PI changes to track changes in $\mathrm{Cl}$ and SVI}

The changes in PI and the changes in CI were correlated when considering all interventions (PLR in 72 patients and volume expansion in 27 patients $)(r=$ $0.63, p<0.0001$, concordance rate $=73 \%$, Fig. 4) or PLR only $(r=0.64, p<0.0001)$. This was also the case for the changes in PI and the changes in SVI when considering all interventions $(r=0.26, p=0.02$, concordance rate $=59 \%)$ or PLR only $(r=0.33, p<0.005$, concordance rate $=63 \%$ ).

\section{Precision of PI measurements}

In included patients, the mean of PI values in measurements performed for assessing the precision was $1.37 \pm$ $0.03 \%$ (in absolute value). In these patients, the coefficient of variation was $3.2 \%$, the precision of PI was $1.2 \%$ and the LSC was $1.6 \%$.

In patients excluded due to the instability of the PI signal, the mean of PI values in measurements performed for assessing the precision was $1.3 \pm 0.2 \%$. In these patients, the precision of PI was $14 \%$, and the LSC was $19 \%$. 


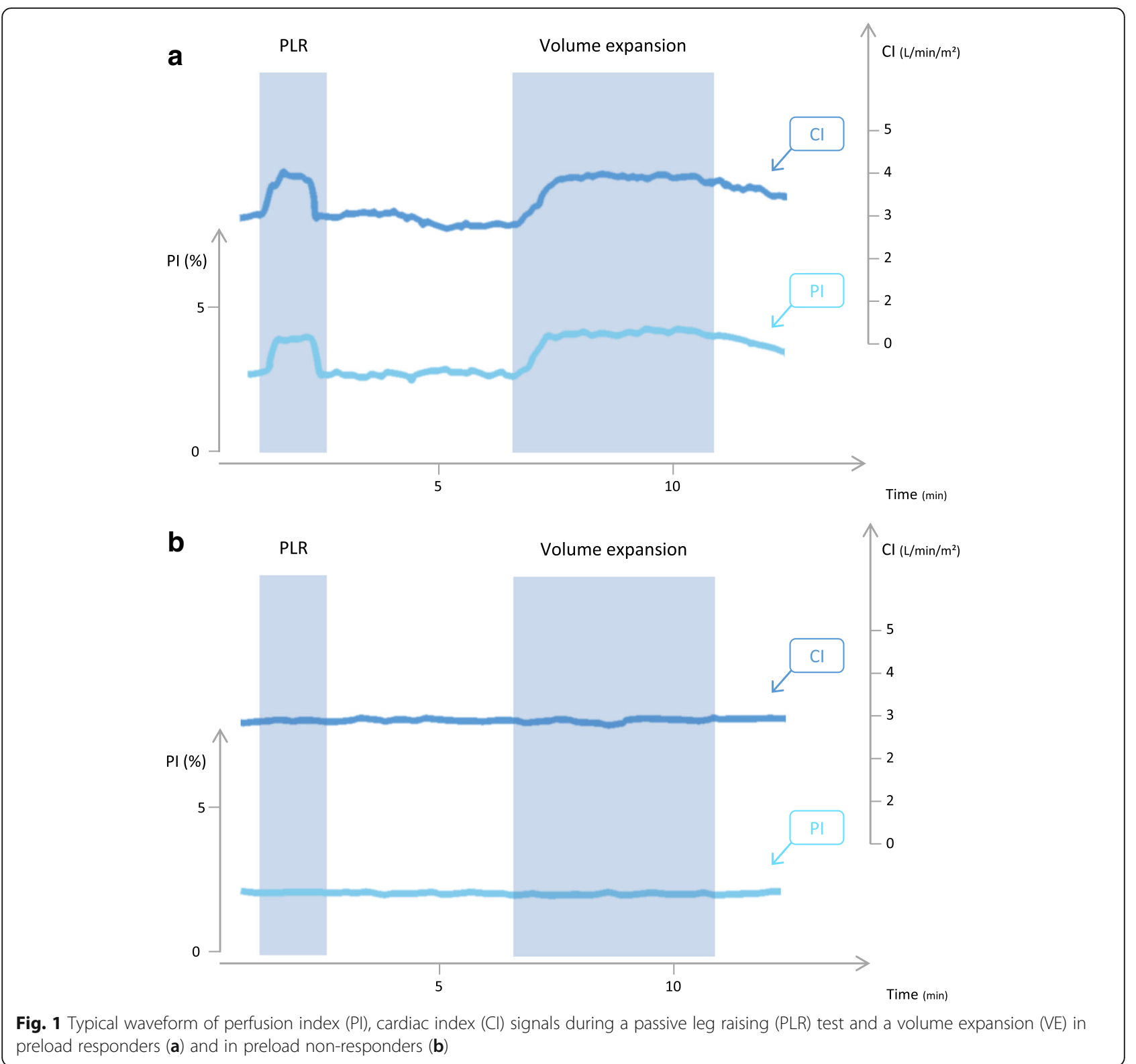

\section{Discussion}

This study suggests that, in patients in whom PI could be measured reliably, the increase in PI detected a positive response of CI to PLR with good accuracy.

The PLR test is an easy and reliable method to predict fluid responsiveness $[9,24]$ which is now accepted in clinical practice $[1,10,25]$. Nevertheless, its main drawback is that, like the fluid challenge $[26,27]$, its effects must be assessed by a direct measurement of $\mathrm{CI}$, which must be precise and able to detect short-term changes with precision [6]. Our study suggests that the changes in PI might be used as a surrogate of the changes in CI during PLR and then could be used to assess preload responsiveness with an acceptable accuracy.
PI has been proposed to reflect the quality of skin perfusion, in particular in anaesthesia [13-15, 28]. Nonetheless, stroke volume should also influence the PI by increasing the arterial blood volume in the finger at each cardiac beat. The relationship between PI and CI cannot be straightforward because it is also influenced by the venous blood flow. A decrease in venous blood flow might cause a stagnation of venous blood in the fingers, an increase in the non-pulsatile component and eventually a decrease in PI independently from the changes in arterial blood flow.

Some studies have suggested that changes in PI reflect changes in CI $[11,29]$ or in the amplitude of arterial pressure [30] in various settings. Desgranges et al. have shown that the changes in PI measured at the forehead 


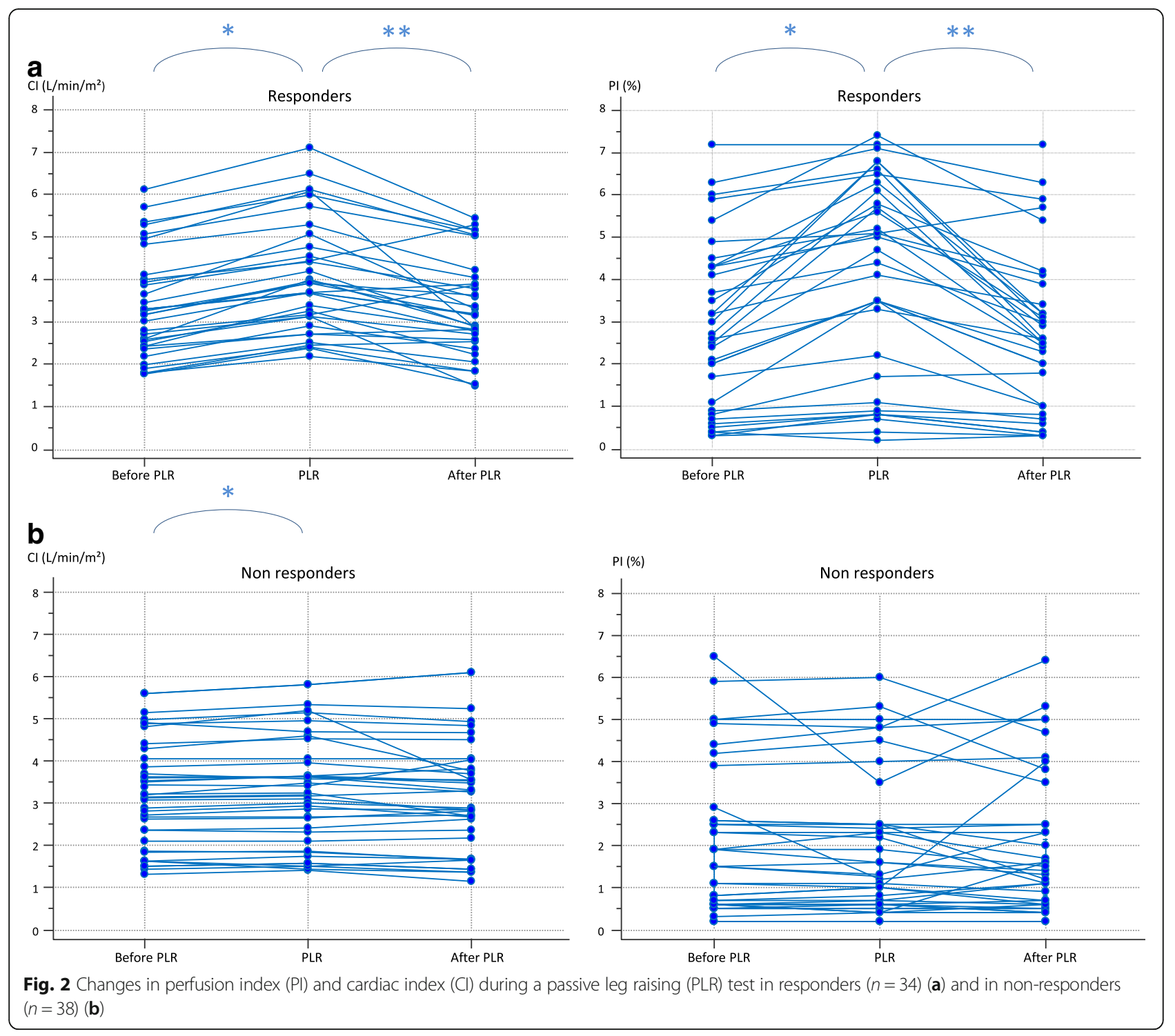

were able to detect the fluid-induced changes in CI with reliable accuracy [31] after induction of anaesthesia. Discrepant results have also been reported [28, 31, 32]. In particular, Broch et al. did not find any significant change in PI during a PLR manoeuvre [28]. Nonetheless, in all these negative studies, the quality of the PI was not mentioned.

Clearly, our results show that PI is not a perfect surrogate of CI. First, the plethysmographic signal was absent in three patients and was unstable in three other ones. Moreover, except in two patients with atrial fibrillation, there was no clear explanation for this instability. Second, the correlation between the changes in PI and changes in CI was not perfect. As stated above, this could be easily explained by the fact that other factors than $\mathrm{CI}$ influence the amplitude of the plethysmographic signal.

One may intuitively think that the low values of PI, the amplitude of which was very variable at baseline, are encountered in patients with the strongest vasoconstriction. Nevertheless, the PI value was not correlated with the dose of norepinephrine, and the PI value at baseline was not different between patients with the highest and the lowest doses of norepinephrine. Even in patients with PI values $<1 \%$, the dose of norepinephrine was not different from that of the other ones. This does not exclude the fact that sepsis-related local hypoperfusion or local vasoconstriction was more pronounced in patients with low PI.

In spite of these limitations, we do think that our data provide new and interesting information. First, we describe a means of assessing the PLR effects that is easy to use, totally non-invasive and available in all patients without any additional cost. Second, in patients with a stable signal, PI was very precise. The precision was much lower than the threshold found to detect a positive PLR test, what means that the PI is a suitable 


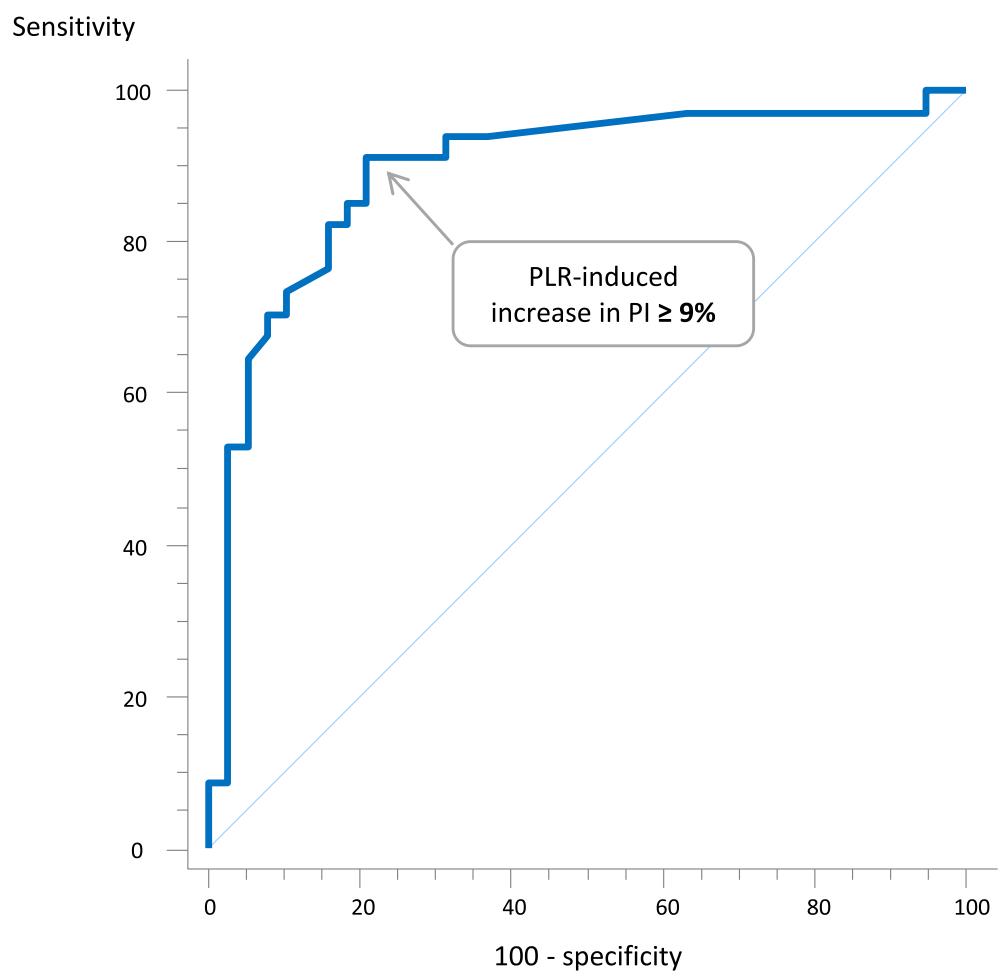

Fig. 3 Area under the receiver operating characteristics curve generated for the detection of a positive passive leg raising (PLR) test by the changes in perfusion index (PI). The Youden index was calculated as sensitivity + specificity -1

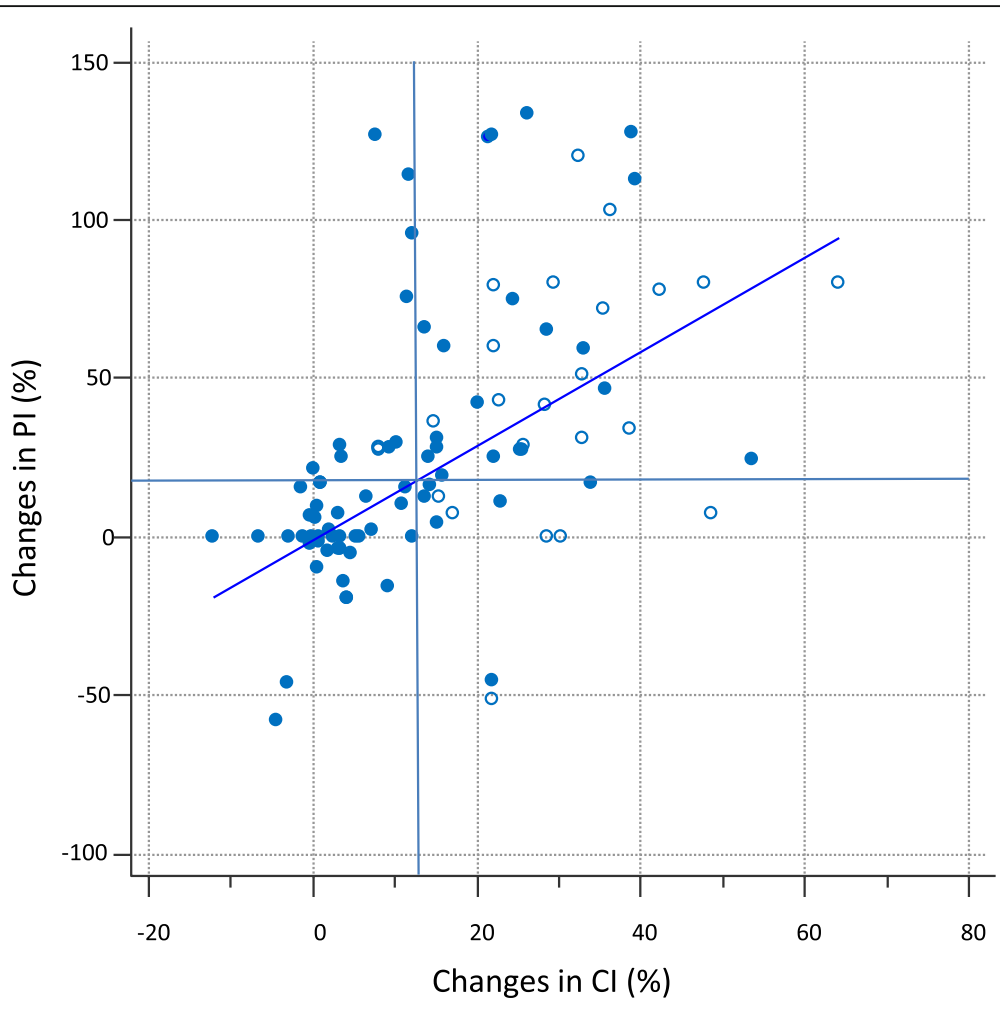

Fig. 4 Correlation between perfusion index (PI) changes and cardiac index (Cl) changes during passive leg raising (O) and volume expansion (O) 
surrogate of CI for this purpose. Third, PI might be used to reflect $\mathrm{CI}$ changes in other clinical circumstances than the PLR test, in which no cardiac output monitoring is available. Fourth, PI might be used even if its value is low $(\leq 1 \%)$.

There are several limitations to our study. First, patients with a negative PLR test did not receive fluids, so that we could not conclude regarding the ability of PLR-induced changes in PI to predict fluid responsiveness. Nevertheless, the reliability of the PLR test should be considered as well established [9]. In the present study, only one patient in whom the PLR test was positive did not respond to fluid administration. In this patient, the PLR-induced increase in CI was close to $10 \%$, and the fluid-induced increase in CI was also close from $15 \%$. Second, we included only critically ill patients while the results regarding PI might differ in other contexts, especially because PI is influenced by skin perfusion, which might for instance be different in the perioperative period [30-32]. Third, we investigated only the PI at the finger level, while its relationship to stroke volume might differ among the site where it is measured [29, 31]. Fourth, we averaged the real-time value of PI over $12 \mathrm{~s}$, which is not performed by the commercial version of the device. Nevertheless, we think this was the only way allowing a comparison with pulse contour analysis-derived CI, which is averaged over $12 \mathrm{~s}$. Moreover, we did not test the "long average" version of the device, which averages PI over $30 \mathrm{~s}$.

\section{Conclusions}

In critically ill patients in whom it could be measured, the changes in PI during PLR test appear a reliable way to assess the haemodynamic effects of the PLR test, and thus to assess preload responsiveness, in a totally non-invasive way. This proof of concept opens the door for further investigations.

\section{Additional file}

Additional file 1: Figure S1. Flowchart $(n=85)$. (PPTX $73 \mathrm{~kb})$

\section{Abbreviations}

Cl: Cardiac index; IAP: Intra-abdominal pressure; LSC: Least significant change; PLR: Passive leg raising; PPV: Pulse pressure variation; ROC: Receiver operating characteristics; SVI: Stroke volume index

\section{Acknowledgements}

Not applicable.

\section{Funding}

No funding

\section{Availability of data and materials}

I agree to the terms of the BioMed Central License Agreement and Open data policy.

\section{Authors' contributions}

$A B$ collected the data, performed the data analysis and drafted the manuscript. J-LT conceived the study, participated in the analysis of the data and wrote the manuscript. FG, FAG, VG, LG and NA contributed to the data recording. CR supervised the study. XM conceived the study, supervised the data analysis and manuscript writing, and coordinated the study. All authors read and approved the final manuscript.

\section{Ethics approval and consent to participate}

- Information and consent obtained for each patient

- Name of the ethics committee that approved the study and the committee's reference number: Comité pour la Protection des Personnes, Ile-de-France VII. Trial registration ID RCB: 2016-A00959-42. Registered 27 June 2016. The patients were included prospectively.

\section{Consent for publication}

All patients of the manuscript have read and agreed to its content and are accountable for all aspects of the accuracy and integrity of the manuscript. The data were used anonymously.

\section{Competing interests}

$J-L$ TEBOUL and X MONNET are members of the Medical Advisory Board of Pulsion Medical Systems. J-L TEBOUL and X MONNET gave lectures for Masimo. The other authors declare that they have no competing interests. No financial support.

\section{Publisher's Note}

Springer Nature remains neutral with regard to jurisdictional claims in published maps and institutional affiliations.

Received: 2 August 2018 Accepted: 2 January 2019

Published online: 18 January 2019

\section{References}

1. Monnet X, Marik PE, Teboul J-L. Prediction of fluid responsiveness: an update. Ann Intensive Care. 2016:6(1):111.

2. Vincent J-L, Sakr Y, Sprung CL, Ranieri VM, Reinhart K, Gerlach H, et al. Sepsis in European intensive care units: results of the SOAP study. Crit Care Med. 2006;34(2):344-53.

3. Acheampong A, Vincent J-L. A positive fluid balance is an independent prognostic factor in patients with sepsis. Crit Care Lond Engl. 2015;19:251.

4. National Heart, Lung, and Blood Institute Acute Respiratory Distress Syndrome (ARDS) Clinical Trials Network, Wiedemann HP, Wheeler AP, Bernard GR, Thompson BT, Hayden D, et al. Comparison of two fluid-management strategies in acute lung injury. N Engl J Med. 2006;354(24):2564-75.

5. Jozwiak M, Silva S, Persichini R, Anguel N, Osman D, Richard C, et al. Extravascular lung water is an independent prognostic factor in patients with acute respiratory distress syndrome. Crit Care Med. 2013;41(2):472-80.

6. Monnet $X$, Teboul J-L. Passive leg raising: five rules, not a drop of fluid! Crit Care Lond Engl. 2015;19:18.

7. Guérin L, Teboul J-L, Persichini R, Dres M, Richard C, Monnet X. Effects of passive leg raising and volume expansion on mean systemic pressure and venous return in shock in humans. Crit Care Lond Engl. 2015;19:411.

8. Monnet X, Rienzo M, Osman D, Anguel N, Richard C, Pinsky MR, et al. Passive leg raising predicts fluid responsiveness in the critically ill. Crit Care Med. 2006:34(5):1402-7.

9. Monnet $X$, Marik P, Teboul J-L. Passive leg raising for predicting fluid responsiveness: a systematic review and meta-analysis. Intensive Care Med. 2016;42(12):1935-47.

10. Cherpanath TGV, Hirsch A, Geerts BF, Lagrand WK, Leeflang MM, Schultz MJ, et al. Predicting Fluid Responsiveness by Passive Leg Raising: A Systematic Review and Meta-Analysis of 23 Clinical Trials. Crit Care Med. 2016;44(5): 981-91.

11. Lima AP, Beelen P, Bakker J. Use of a peripheral perfusion index derived from the pulse oximetry signal as a noninvasive indicator of perfusion. Crit Care Med. 2002;30(6):1210-3.

12. Goldman JM, Petterson MT, Kopotic RJ, Barker SJ. Masimo signal extraction pulse oximetry. J Clin Monit Comput. 2000;16(7):475-83.

13. Lima A, Bakker J. Noninvasive monitoring of peripheral perfusion. Intensive Care Med. 2005 Oct;31(10):1316-26. 
14. van Genderen ME, Bartels SA, Lima A, Bezemer R, Ince C, Bakker J, et al. Peripheral perfusion index as an early predictor for central hypovolemia in awake healthy volunteers. Anesth Analg. 2013;116(2):351-6.

15. Toyama S, Kakumoto M, Morioka M, Matsuoka K, Omatsu H, Tagaito Y, et al. Perfusion index derived from a pulse oximeter can predict the incidence of hypotension during spinal anaesthesia for Caesarean delivery. Br J Anaesth. 2013;111(2):235-41.

16. Kirkpatrick AW, Roberts DJ, De Waele J, Jaeschke R, Malbrain MLNG, De Keulenaer B, et al. Intra-abdominal hypertension and the abdominal compartment syndrome: updated consensus definitions and clinical practice guidelines from the World Society of the Abdominal Compartment Syndrome. Intensive Care Med. 2013;39(7):1190-206.

17. Jozwiak M, Monnet X, Teboul J-L. Pressure Waveform Analysis. Anesth Analg. 2017.

18. Monnet X, Teboul J-L. Transpulmonary thermodilution: advantages and limits. Crit Care Lond Engl. 2017;21(1):147.

19. Monnet X, Persichini R, Ktari M, Jozwiak M, Richard C, Teboul J-L. Precision of the transpulmonary thermodilution measurements. Crit Care. 2011;15(4):R204.

20. Monnet X, Cipriani F, Camous L, Sentenac P, Dres M, Krastinova E, et al. The passive leg raising test to guide fluid removal in critically ill patients. Ann Intensive Care. 2016;6(1):46.

21. Cecconi M, Rhodes A. Validation of continuous cardiac output technologies: consensus still awaited. Crit Care Lond Engl. 2009;13(3):159.

22. Hapfelmeier A, Cecconi M, Saugel B. Cardiac output method comparison studies: the relation of the precision of agreement and the precision of method. J Clin Monit Comput. 2016:30(2):149-55.

23. Hanley JA, McNeil BJ. The meaning and use of the area under a receiver operating characteristic (ROC) curve. Radiology. 1982;143(1):29-36.

24. Mesquida J, Gruartmoner G, Ferrer R. Passive leg raising for assessment of volume responsiveness: a review. Curr Opin Crit Care. 2017;23(3):237-43.

25. Cecconi M, De Backer D, Antonelli M, Beale R, Bakker J, Hofer C, et al. Consensus on circulatory shock and hemodynamic monitoring. Task force of the European Society of Intensive Care Medicine. Intensive Care Med. 2014;40(12):1795-815.

26. Monnet X, Letierce A, Hamzaoui O, Chemla D, Anquel N, Osman D, et al. Arterial pressure allows monitoring the changes in cardiac output induced by volume expansion but not by norepinephrine. Crit Care Med. 2011;39(6): 1394-9.

27. Pierrakos C, Velissaris D, Scolletta S, Heenen S, De Backer D, Vincent J-L. Can changes in arterial pressure be used to detect changes in cardiac index during fluid challenge in patients with septic shock? Intensive Care Med. 2012;38(3):422-8.

28. Broch O, Bein B, Gruenewald M, Höcker J, Schöttler J, Meybohm P, et al. Accuracy of the pleth variability index to predict fluid responsiveness depends on the perfusion index. Acta Anaesthesiol Scand. 2011;55(6):686-93.

29. McGrath SP, Ryan KL, Wendelken SM, Rickards CA, Convertino VA. Pulse oximeter plethysmographic waveform changes in awake, spontaneously breathing, hypovolemic volunteers. Anesth Analg. 2011;112(2):368-74.

30. Cannesson M, Delannoy B, Morand A, Rosamel P, Attof Y, Bastien O, et al. Does the Pleth variability index indicate the respiratory-induced variation in the plethysmogram and arterial pressure waveforms? Anesth Analg. 2008; 106(4):1189-94.

31. Desgranges F-P, Desebbe O, Ghazouani A, Gilbert K, Keller G, Chiari P, et al Influence of the site of measurement on the ability of plethysmographic variability index to predict fluid responsiveness. Br J Anaesth. 2011;107(3): $329-35$

32. Cannesson M, Desebbe O, Rosamel P, Delannoy B, Robin J, Bastien O, et al. Pleth variability index to monitor the respiratory variations in the pulse oximeter plethysmographic waveform amplitude and predict fluid responsiveness in the operating theatre. Br J Anaesth. 2008;101(2):200-6

Ready to submit your research? Choose BMC and benefit from:

- fast, convenient online submission

- thorough peer review by experienced researchers in your field

- rapid publication on acceptance

- support for research data, including large and complex data types

- gold Open Access which fosters wider collaboration and increased citations

- maximum visibility for your research: over $100 \mathrm{M}$ website views per year

At $\mathrm{BMC}$, research is always in progress.

Learn more biomedcentral.com/submissions 